\title{
La disolución de los límites en el espacio religioso.
La Capilla del Bosque de Heikki
} y Kaija Siren

\author{
The dissolution of the boundaries in the religious space. \\ The Forest Chapel of Heikki and Kaija Siren
}

\section{Resumen}

Autores:

Nieves Fernández Villalobos* nfvillalobos@yahoo.es

Andrés Jiménez Sanz** andresjimenezsanz@gmail.com

*Universidad de Valladolid **Investigador independiente

España

Recibido: 30/Jul/2020 Aceptado: 23/Nov/2020
$\mathbf{L}$ os arquitectos finlandeses Heikki y Kaija Siren levantaron en 1957 una pequeña capilla en el Campus Tecnológico de Otaniemi, expresando los vínculos nórdicos con el paisaje bajo las consignas del racionalismo naturalista. Crearon un espacio de intimidad, en medio de la naturaleza, donde los fieles pudieran ahondar en su interior. La capilla que Erik Bryggman había construido anteriormente para el Cementerio de Turku, con su paño de vidrio lateral, supuso una referencia imprescindible para la obra de los Siren. En el desarrollo de su proyecto fueron modificando la apertura hacia el paisaje hasta llegar a una secuencia espacial, repleta de matices, que comienza y acaba en el bosque. La investigación profundiza en esta obra maestra de la arquitectura moderna de posguerra y la relevancia que ha tenido en la creación de otros espacios litúrgicos coetáneos y posteriores, hasta llegar a las contemporáneas Capillas Vaticanas, donde el espacio religioso se vincula también singularmente con el lugar, disolviendo sus límites de forma significativa.

Palabras clave: espacio religioso; límites; Siren; Bryggman; Capillas Vaticanas.

\section{Abstract:}

Finnish architects, Heikki and Kaija Siren, built a small chapel in 1957 on the Otaniemi Technology Campus, expressing Nordic linkage to the landscape, under the values of naturalistic rationalism. They created a space of intimacy, in the middle of nature, where the faithful could delve inside. The chapel that Erik Bryggman had previously built for the Turku Cemetery, with its side glass screen, served as an essential reference, and the Sirens, in the development of their project, gradually modified the opening to the landscape until they reached a full spatial sequence, which begins and ends in the forest. The research deepens into this masterpiece of modern post-war architecture and the relevance it has had in the creation of other contemporary and later liturgical spaces, until reaching contemporary Vatican chapels, where the religious space is also singularly linked to the place, dissolving its limits significantly.

Keywords: religious space; boundaries; Siren; Bryggman; Vatican Chapels. 


\section{Introducción.}

\section{La esencia atemporal de la capilla finlandesa}

En el año 2009, The Intenational Carlo Scarpa Prize for Gardens recaía sobre la pequeña Capilla de Otaniemi, en Helsinki, construida en 1957 por los arquitectos Kaija (1920-2001) y Heikki Siren (1918-2013). El prestigioso galardón, instaurado por la Fundación Benetton Studi Ricerche, tiene el objetivo de estudiar y difundir una obra que destaque por su intervención en el paisaje en el que se inserta. En principio, podría resultar curioso que un premio de paisaje recayera sobre la pequeña capilla finlandesa, que destaca por su singular calidad tectónica y por su actitud notablemente respetuosa respecto al paisaje, dejándolo intacto en su implantación. Pero es precisamente el entorno natural el que da sentido a este proyecto; únicamente el bosque justifica esta intervención que ejemplifica extraordinariamente la disolución de los límites entre arquitectura y paisaje. El jurado destacaba (Luciani y Puppi, 2009, p. 8):

La magistral esencia y transparencia de esta obra arquitectónica devuelve a la naturaleza la tarea de mostrar lo sagrado, y da sentido y medida a un espacio de experiencia educativa, espiritual y social, cultural y musical, para toda la comunidad de familias y estudiantes. Naturaleza, arquitectura y sociedad se encuentran así en este admirable crisol de forma y vida...

Esta obra ya había sido ampliamente difundida durante los años posteriores a su construcción, a través de algunas revistas. Con este homenaje, la mirada internacional pretendía llamar nuevamente la atención sobre la capilla. El artículo se propone explicar su génesis y reflexionar sobre las decisiones claves que dirigieron el desarrollo del proyecto, así como ahondar en el papel simbólico que ha jugado en la comprensión de lo sagrado, estableciendo nuevas maneras de entender el espacio religioso desde la disolución de los límites, como manifiestan también algunas de las iglesias que en ella se inspiran.

\section{Otaniemi. Naturaleza y Memoria}

El bosque constituye un lugar cotidiano, y a la vez singular para cualquier finlandés. Russell Walden escribe sobre ese bosque que late en muchas construcciones de Finlandia en el libro Finish Harvest, y de manera muy especial en la Capilla de la Universidad Técnica de Otaniemi. Sus autores, Heikki y Kaija Siren, fueron un matrimonio de arquitectos de larga trayectoria profesional, cuya obra atravesó por etapas diferenciadas. Durante sus primeros años trabajaron en Helsinki con el padre de Heikki, el arquitecto Johan Sigfrid Siren, cuyas obras destacaban por expresar la estabilidad monumental del clasicismo. La pareja abrió en 1949 su propio estudio en Helsinki $y$, aunque posteriormente incorporaron nuevas sedes en Austria, Finlandia y Suiza, su obra inicial mantiene un constante diálogo con la naturaleza y confiere a cada edificio un carácter intimista, que parece diluirse en obras posteriores, de mayor escala, realizadas en otros países.

En 1949 se celebró un concurso con el objetivo de diseñar la urbanización de Otaniemi, y el proyecto realizado por los Siren con Aarne Nervi quedó en segundo lugar. El primer premio recayó en Alvar y Aino Aalto, con el lema Ave Alma Mater. Alvar Aalto había comenzado a apreciar el modelo anglosajón de campus universitario, debido a sus numerosas estancias en Massachusetts como profesor invitado, y así lo demostró en Otaniemi. Su plan comprendía un conjunto de edificios jerarquizados topográficamente, que se articulaban mediante espacios abiertos y de circulación. De la mano del finlandés fluyeron sus características formas en abanico y serpentinas, generando espacios que llevaban a una mística relación con la naturaleza. El lugar invitaba a ello de una manera muy especial: Espoo era un área de gran belleza natural, relativamente virgen, y Otaniemi, situado en las orillas de la Bahía de Laajalahti, constituía un atractivo paisaje de rocas de granito, pinos y abedules. Así, el plan de Aalto ponía su acento en la preservación de las condiciones naturales.

Ya en 1953, el renombrado arquitecto tenía el encargo oficial de realizar el proyecto de la edificación principal. Esta, la única desarrollada completamente por él, consistía en un juego equilibrado de volúmenes en ladrillo rojo oscuro, granito negro y cobre que, especialmente en el Aula Magna, muestra la influencia de sus viajes a la Europa Clásica, y en concreto de las ruinas del Teatro de Delphos, visitadas en 1953 (Jové, 2003, pp. 253- 291). ${ }^{1}$ Poco después, otros dos matrimonios de arquitectos comenzaron a trabajar en Otaniemi, dentro del plan general concebido por Aalto (Figura 1). Una de esas parejas la conformaba los Siren, a quienes les encargaron primero una sauna para estudiantes, un restaurante y tres bloques residenciales. Fruto de un concurso, realizarían después la pequeña capilla. Entre 1961 y 1966, y también como resultado de un concurso en el que Aalto formaba parte del jurado, los arquitectos Reima y Raili Pietilä llevaron a cabo la Sede del Sindicato de Estudiantes, conocido como Dipoli. Ambos equipos integraron sus edificios en el paisaje, aunque cada uno siguiendo su propio estilo e imprimiendo el carácter finlandés a través de distintas expresiones. Con el lema "la marcha de los hombres de las cavernas", Dipoli constituye un espacio plástico que convierte en propias las formas del lugar: las rotundas cubiertas del edificio y la línea quebrada de la fachada responden al perfil abrupto circundante (Connah, 1998, p. 451). Las rocas, dispersas en el paisaje, llegan incluso a irrumpir en el interior. Conformando una singular metamorfosis entre la regularidad y la forma libre, Dípoli se pronuncia contundente y sus espacios escultóricos, de huella expresionista, se presentan como vigorosas cavernas.

\footnotetext{
${ }^{1}$ Kaija Siren ha relatado que, en el transcurso de una conferencia impartida por Aalto, mientras se consideraba la realización de los edificios residenciales, en un momento en el que creía el micrófono apagado, se le pudieron escuchar las palabras: "Dejad que los hagan los jóvenes" (Fax que envía Kaija Siren a Russell Walden, el 17 de Mayo de 1994, tal y como relata el autor en el citado libro).
} 

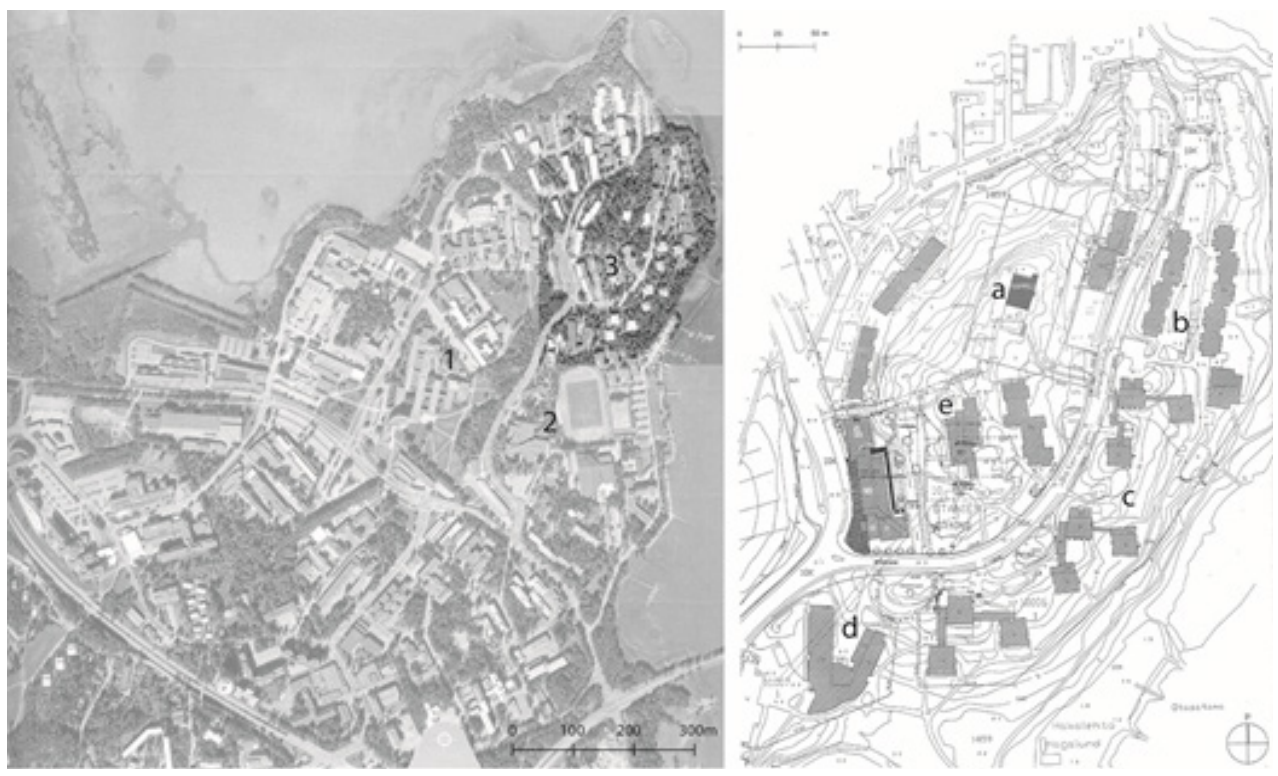

Figura 1: Fotografía aérea de la península de Otaniemi, señalados los edificios principales desarrollados por Alvar Aalto para el Campus (1), Dipoli de Reima y Reili Pietilä (2) y el área residencial de estudiantes, con la capilla de Heikki y Kaija Siren (3). A la derecha, zona residencial del campus, con la capilla (a), los edificios residenciales de Martti Melakari (b), H. y K. Siren (c) y Aalto (d) y the Servi Cottage de M. Melakari y H. y K. Siren (e)

Fuente: National Land Survey of Finland, 2006, (Luciani y Puppi, 2009)

Las obras de los Siren en Otaniemi se relacionaban con el paisaje de forma muy distinta: si la escultural cueva de Pietilä parecía pretender eclipsar los edificios de Aalto, Heikki Siren, junto con Martti Melakari, proyectaron silenciosamente sus edificios conviviendo con los árboles de la zona norte de Otaniemi. El restaurante, Servin Mökki, concebido en 1952 como un espacio unitario, partía de dos volúmenes desplazados entre sí. A pesar de que su resolución exterior reflejaba una lógica constructiva poco clara, el espacio diáfano del interior, construido con pórticos de madera, presumía de un atractivo ritmo estructural y espacial, así como de una amplia pared de vidrio en orientación sur, que parecía anticipar el proyecto del santuario (Royo, 2014, p. 106).

Encontramos en Otaniemi, en las intervenciones de Aalto, los Pietilä y los Siren, las tres maneras de entender la relación entre naturaleza y arquitectura que recoge Fabrizio Tucci en su artículo "Naturaleza y Memoria en la Arquitectura Finlandesa" (Tucci, 1994, pp. 61-67). Como este autor explica, existe en todo hombre un sentimiento, más o menos latente, de amor y pertenencia a la naturaleza, que la arquitectura es capaz de expresar mediante distintos procedimientos. El primero, "e racionalismo naturalista", emplea formas puras y simples que dan la oportunidad de hacer sentir al hombre la diversidad del ambiente natural. Dentro de esta postura el autor señala a Mies van der Rohe, los Siren, y Tadao Ando. El segundo planteamiento, "la arquitectura orgánica", protagonizada por Frank Lloyd Wright y los Pietilä, pretende dar continuidad a la naturaleza, expresarse con sus formas y participar de ella. Una tercera posición, encabezada por Erik Gunnar Asplund, Sigurd Lewerentz y Alvar Aalto, realiza la arquitectura desde una objetividad y pureza fascinante, desviándose de la informalidad presente en la naturaleza. Los Siren defienden así la profundidad de la primera de las tres posiciones:
El amor del arquitecto por la naturaleza, su compromiso con ella es un intento de organizar las relaciones entre el espacio, la apertura, la luz y la sombra para permitir al hombre en medio de la arquitectura experimentar la diversidad de la naturaleza y la variedad de las estaciones en un entorno integrado con ella y sin violarla. No significa la adopción de "formas propias de la naturaleza" en arquitectura... A menudo, los mejores ejemplos de buena adaptación a la naturaleza se han encontrado en la interacción de las colonias clásicas y sus restos, tras la proporción perfecta y la expresión sobria de las formas y lo pletórico que surge de la naturaleza no domesticada. Un buen ejemplo es la casa Farnsworth de Mies van der Rohe, donde este efecto de contrapunto se produce brillantemente. (Siren, 2009, p. 26).

\section{Origen y evolución de la Capilla del Bosque}

La Casa Farnsworth se finalizó en 1950 y, jLa Casa Farnsworth se finalizó en 1950 y, justo después, con los objetivos de "contraponer las formas, organizar los espacios, las luces y las sombras", e integrarse en la naturaleza, los Siren comenzaron a proyectar la que sería su obra más reconocida. Pero el proceso no fue inmediato. La larga lucha para realizar esta obra empezó en 1951, cuando la pareja recibió instrucciones de proyectar "una capilla luterana para el Campus". Aalto no había incluido inicialmente un centro religioso dentro de su plan de ordenación, sino que su demanda nació de los primeros grupos estudiantiles, a los que se les había asignado las viviendas construidas para los atletas de los juegos Olímpicos de 1952. Organizados en el Ristin Kilta (Cofradía de la Cruz), los estudiantes consiguieron financiación y encargaron la construcción del templo a los arquitectos. 


\subsection{La lección de Bryggmann. Simbolismo y narración}

La primera propuesta de los Siren planteaba una construcción de ladrillo que partía de un cuadrado de trece metros y medio. En esta manera de concebir e proyecto los arquitectos se acercaron al pensamiento de Kahn, introduciendo apriorísticamente figuras geométricas que anteceden a la función, y considerando posteriormente las actividades que se van a desarrollar como verificación de un acto intuitivo. Kahn distingue la capacidad estática que proporciona el cuadrado, frente al dinamismo que sugieren las formas longitudinales (Gil, 1999, p. 92), de modo que los Siren optaron inicialmente por la disposición asimétrica de una planta cuadrada, buscando transmitir meditación y quietud. Se aprovechó el desnivel existente al sur de la parcela para crear un espacio inferior independiente, y se señaló el acceso en la parte superior con un pequeño volumen cúbico descentrado: un singular elemento que sale de la estricta geometría del templo para acoger a los fieles. El espacio interior se intuye puro y honesto, destacando exclusivamente una vidriera que se abre al oeste: única fuente de luz de la capilla, que permite la comunicación directa con los árboles del bosque (Figura 2).

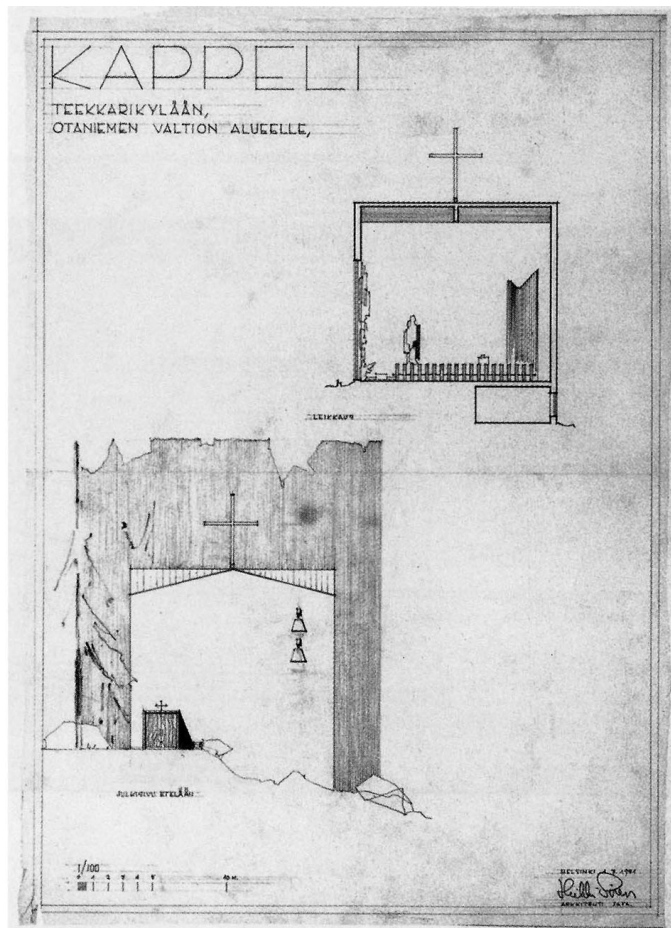

Figura 2: Heikki Siren: Propuesta inicial para la Capilla de Otaniemi, 1951

Fuente: (Ibáñez (coord.) 2005, pp. 38- 39)

Esa primera propuesta de los Siren es claramente deudora de la Capilla que Erik Bryggman había construido, entre 1938 y 1941, para el Cementerio de Turku; si bien en esta, se empleó la longitudinalidad para dar protagonismo a los recorridos. Como lema para su proyecto, Bryggman escogió una frase de Baruch de Spinoza $^{2}$, sub specie aeternitatis (desde la perspectiva

${ }^{2}$ Baruch de Spinoza fue un racionalista holandés del siglo XVII, cuya filosofía parte de la identificación de Dios con la naturaleza (Deus sive natura), hasta convertirse en el mayor exponente moderno del panteísmo. de la eternidad), haciendo referencia a la dimensión cósmica del espacio y el tiempo, sin perder de vista la futilidad humana (Bennet, 1991). El autor incorporó esa expresión en los planos y croquis iniciales, como puede observarse en uno de sus sugerentes perspectivas del espacio interior. La mano de Bryggman revela, con un expresivo trazo multidireccional, la importante labor que la luz y la escala ejercen en su capilla, repleta de profundas alegorías (Figura 3)
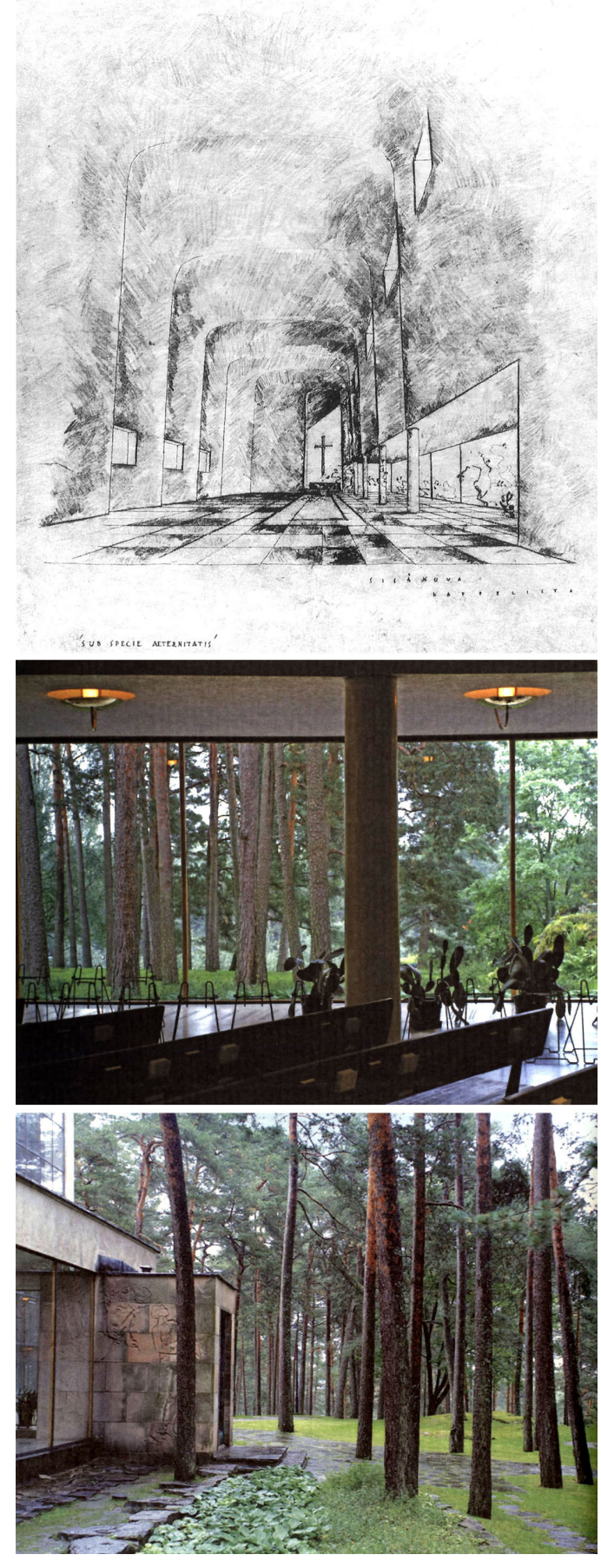

Figura 3: E. Bryggman: Capilla para el Cementerio de Turku, 1938-1941

Fuente: Nikula, 1991, p. 214 (a) Luciani y Puppi, 2009 (b, c) 
La imagen se mantuvo prácticamente intacta en el proyecto construido, alterándose tan solo las proporciones y forma del altar. Los elementos de la capilla responden a las distintas fases del ritual. Tan solo una nave, cubierta con una bóveda rebajada, conforma el cuerpo de la iglesia e imita la disposición asimétrica del pórtico; permite el acceso de los fieles por uno de sus extremos, dejando los bancos a su izquierda simbólicamente girados. A la derecha, la nave permanece flanqueada por cinco columnas, que dirigen las miradas hacia un paño de vidrio lateral. Un prisma de piedra irrumpe al final de ese extraño cuerpo más bajo y permeable, configurando una simbólica puerta. La percepción de la naturaleza, que entra a formar parte del espacio interior, completa una situación de tensión y dinamismo, provocada por la singular forma de la nave y por la disociación entre la concepción simétrica exterior y asimétrica interior. El núcleo del altar está desplazado, y sobre él cae teatralmente un intenso rayo de luz que llega desde una fuente oculta, tras la "Puerta de la Esperanza". Bryggman provoca una lectura simbólica mediante la presencia del exterior en el interior, desdibujando los límites: el bosque que se ve desde el lugar del duelo representa la esperanza en la resurrección. Todo el camino recorrido, iniciado con el procesional ascenso desde el cementerio y seguido por la puerta principal hasta la que se encuentra al fondo del altar, escenifica el tránsito feliz después de la muerte. A pesar de ser una propuesta contenida, suspendida entre dos tradiciones, con evidente contraste entre su retórico aspecto exterior y la austera desnudez del espacio interior, el edificio de Bryggman propone nuevas cuestiones a través de su dinamismo y narrativa simbólica; aspectos que, sin duda, tuvieron numerosas repercusiones (Gil, 1999, p. 44).

\subsection{Pasado y presente del espíritu finlandés}

Los estudiantes de Otaniemi, por lo general, se mostraron satisfechos ante esa primera capilla ideada por Heikki Siren, pero algunos se opusieron al proyecto, por lo que el propio arquitecto propuso que se realizara un concurso. Así, el 5 de enero de 1954, cuarenta arquitectos presentaron sus propuestas a un jurado en el que participó Aulis Blomstedt. En mayo de 1954 se publicaron los tres primeros premios, que estaban fuertemente influenciados por la Capilla de Bryggman. ${ }^{3}$ El proyecto Altaír (Altar) de los Siren, una arriesgada evolución de su idea inicial, no obtuvo ningún premio por exceder el área reglamentaria. Pero ninguna de las propuestas premiadas satisfacía completamente al jurado, por lo que reconsideraron el proyecto de los Siren y estipularon que era el único que tenía "la poesía de la auténtica arquitectura". Así, gracias a la persuasiva dialéctica de Blomstedt, la realización de la capilla fue encomendada al joven matrimonio (Walden, 1998, p. 44).

Las obras de los Siren nacen de la búsqueda de soluciones funcionales a partir de elementos mínimos, algo que Heikki había practicado con la observación y diseño de veleros (Quantril, 2005, p. 111). ${ }^{4}$ Para la realización de la Capilla, los arquitectos habían intentado fusionar los elementos racionales y los místicos. La

\footnotetext{
${ }^{3}$ El primer premio fue concedido a Erkki Pasanen y Kauko Tiihonen, el segundo a Martikainen-Ypyä, y el tercero a Pentti Petäjä y Esko Hyvärinen. (Arkkitehti Arkitekten, May, 1954, pp. 35-38).
}

solución final perseguiría abrazar singularmente el pasado y el presente del espíritu finlandés. Estudiaron la arquitectura tradicional finlandesa, los pueblos rurales y sus iglesias del período tardomedieval, en los que reinaba el orden y una inmensa claridad racional (Figura 4). Eran edificios expresivos realizados en piedra gris, con tejados de madera de fuertes pendientes, que incluían patios tapiados con volumétricos campanarios exentos y coloridos frescos en el interior. Bajo una mirada moderna, los Siren interpretarían en su capilla la lección obtenida de las iglesias tradicionales: el orden y la geometría subyace en todo el proyecto, la cubierta adquiere una fuerte pendiente y se enfoca hacia la cruz final, y el patio de acceso aloja un campanario exento y volumétrico, aunque desmaterializado en su solución constructiva.

El reconocido arquitecto finlandés Juhani Pallasmaa ha destacado el papel de esas antiguas iglesias como una "imagen embrionaria de la religión", que predispone sensorialmente a la experiencia religiosa. ${ }^{5}$ De esa tradición, los Siren aprendieron a fusionar las formas puras con materiales naturales; unas formas que debían responder a la función y vincularse inherentemente al bosque. Los Siren entendían la belleza del paisaje circundante como una imagen de la humanidad (Walden, 1998, p. 17). Así, utilizaron este fragmento de la poesía de Eino Leino para explicar lo que siente el alma finlandesa:

I do not laugh or grieve, or sigh,

the forest's darkness breathes nearby,

the red of clouds where day sinks deep,

the blue of windy hills asleep,

the twinflower's scent, the water's shade

of these my heart's own song is made.

Eino Leino conseguía hacer conscientes a los finlandeses de la inmensa herencia natural que tenían, rebosante de tonalidades y bañada por la difusa luz nórdica. Los Siren quisieron trasladar esos ricos matices a la pequeña Capilla. Así, aunando la sensibilidad de la tradición con la simplicidad técnica y material, fueron definiendo el lugar.

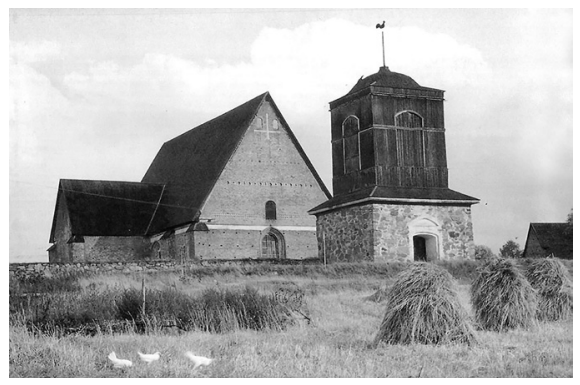

Figura 4: Iglesia de la Santa Cruz en Hattula, S. XIV-XV Fuente: Walden, 1998, p. 78

${ }^{4}$ Heikki Siren diseñó varios veleros a modo de ejercicio funcionalista, por ser objetos en los que la forma afecta a la función de manera muy directa.

${ }^{5}$ Pallasmaa describe su primera visita a una de estas iglesias, con 6 años: "todavía tengo vívidos recuerdos sensoriales del imponente frontón de la iglesia de piedra de Tuulos en la penumbra, su techo negro tan alto como una montaña, los enormes muros de piedra, e interior tipo caverna, la luz en la vela parpadeante, el olor a cera y parafina, el eco de la predicación del pastor y el canto de himnos en la vasta inmensidad de arriba" (Pallasmaa, 2009, p. 94).

6 "No me río o me lamento, o suspiro, la oscuridad del bosque respira en la proximidad, el rojo de las nubes donde el sol se hunde profundamente, el azul de las tempestuosas colinas se queda dormido, la fragancia de las flores, la sombra del agua, con todo esto, está hecha la canción de mi alma." Eino Leino (1878-1926), Nocturne, 1903 (Traducción del autor). 


\subsection{La trascendencia de la progresión espacial}

El solar que se presentó al concurso afectaba a la manera en que los Siren habían comenzado a afrontar el proyecto. Era estrecho y largo, situado en un claro del bosque. Heikki admiraba el delicado vínculo del hombre con la naturaleza presente en la arquitectura japonesa:

\begin{abstract}
El amor del arquitecto por la naturaleza, su compromiso con ella, es un intento de organizar las relaciones entre el espacio, las aperturas, la luz y la sombra, para permitir al hombre en medio del paisaje experimentar la diversidad de la naturaleza y la variedad de las estaciones en un entorno integrado con ella y sin violarla... En Japón, este delicado vínculo entre la naturaleza y el hombre tiene una larga relación (Siren, 2009 p. 26).
\end{abstract}

La capilla de Otaniemi, habitando silenciosa entre los árboles, recuerda al recinto Sagrado de Ise, cuyo espacio rectangular era concebido como un vacío en el bosque y se percibía solamente en relación con el fluir del tiempo. La capilla de Otaniemi, entre los árboles, ahonda en significados afines (Espuelas, 1999, pp. 75- 85).

Dentro del área rectangular ofrecida por el concurso, tan solo se podía construir en una pequeña zona, a partir de la cual el terreno iba descendiendo hacia el norte. Por tanto, la orientación y el terreno fueron decisivos en la solución final del proyecto. Frente a la propuesta de planta cuadrada con la que habían comenzado, el solar rectangular invitaba a jugar con los recorridos. Intuitivamente, los arquitectos trazaron dos líneas paralelas de doble ladrillo, que delimitaban los laterales con determinación. Dentro de esta nueva definición rectangular, plantearon una progresión espacial que arrancaba de un patio semicerrado hacia el vestíbulo de entrada y finalizaba en el espacio principal de los fieles (Figura 5).

Pero los arquitectos percibían que la idea no tenía suficiente intensidad, por lo que trabajaron varias noches acompañados de gélidas nieves, replanteándose esa secuencia de espacios. Concibieron así la idea de una pared de cristal en el altar del santuario, con una cruz firme en la nieve. El espacio se metamorfosearía en un objeto de contemplación de la belleza. Lo que faltaba en el primer proyecto era la elaboración de ese concepto: la creencia de la Divinidad del mundo natural. Con este objetivo, los Siren cambiaron por completo el proyecto inicial de su capilla. Mientras que Bryggman había utilizado la transparencia para potenciar el simbolismo de los recorridos, los Siren la emplearían para conseguir la identificación de la Divinidad con el paisaje circundante. Los fieles contemplan los cambios de las estaciones tras la cruz y el tabernáculo. El telón acristalado que enmarca el bosque posibilita sentir con fuerza la naturaleza desde la vivencia de un interior, que así se convierte en sagrado.

Los Siren potenciaron que su proyecto fuera una secuencia de espacios intermedios que se iniciara y finalizara con el bosque. La planta y la sección definen y reconducen gradualmente la simplicidad intimista de este concepto (Figura 6). La obra se ejecutó siguiendo fielmente los planos del concurso, si bien se aumentó la zona previa por motivos funcionales, lo que favorecía la secuencia espacial con una mayor longitudinalidad que se producía como suma de distintos cuadrados (Fernández, 2014, p.227) (Figura 7).

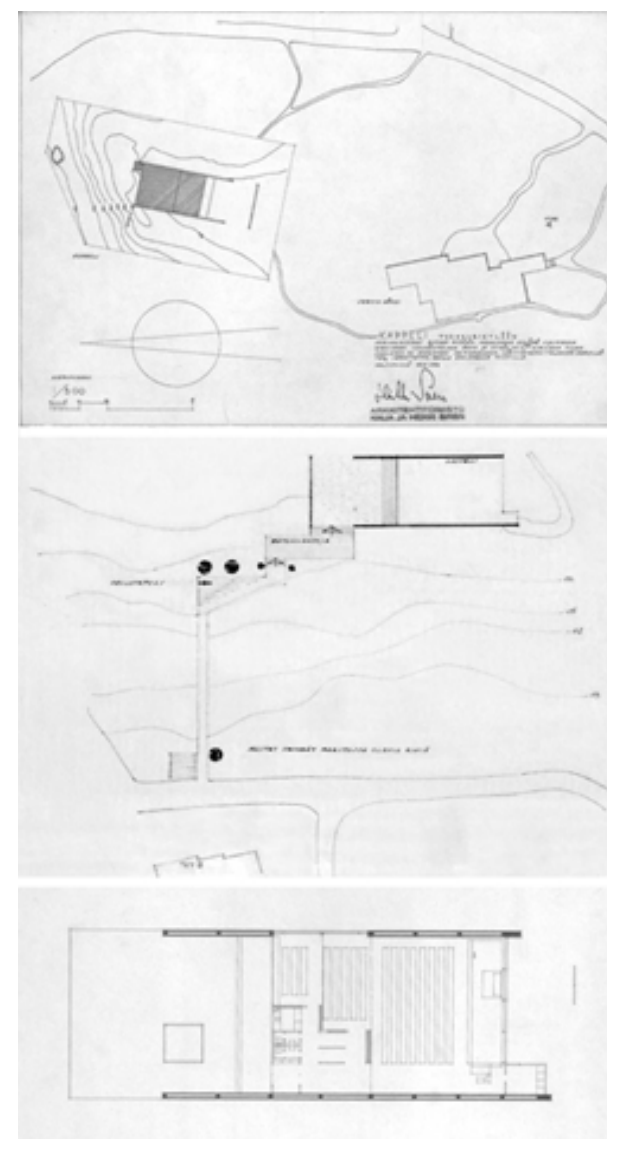

Figura 5: Kaija y Heikki Siren, Capilla de Otaniemi: plano de situación (29/10/1956) / sección longitudinal (1956) / planta del proyecto de reconstrucción de la capilla, tras el incendio (1977)

Fuente: Luciani y Puppi, 2009

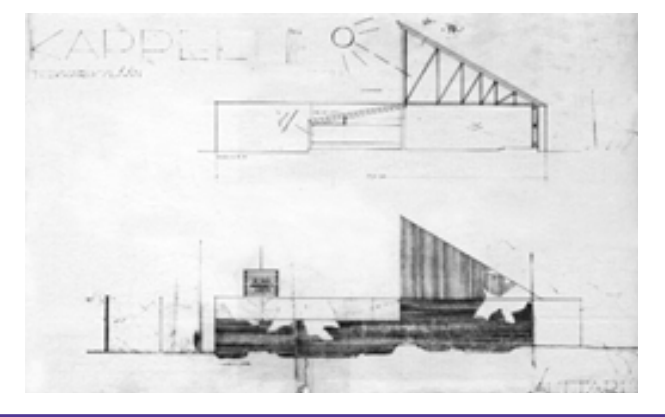

Figura 6: Kaija y Heikki Siren, Capilla de Otaniemi: sección longitudinal del proyecto, en su primera versión, 1956 Fuente: Luciani y Puppi, 2009

El primero es un recinto ligeramente delimitado al que se llega a través de un sendero dibujado entre los árboles del bosque. Cambia la densidad de las sombras y unas pocas escaleras se extienden en el camino, anunciando la capilla. El atrio tenía que ser lo suficientemente cerrado como para recrear en sí mismo los antiguos recintos finlandeses con sus campanarios exentos y, a la vez, lo suficientemente abierto para resumir el mundo natural circundante. Los límites del patio se van desvaneciendo en su cercanía al bosque. Esta manera de ir aligerando el perímetro del recinto, presentando cada muro distinta 


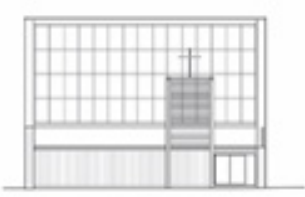

atraso our

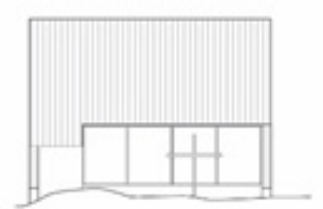

azado norte

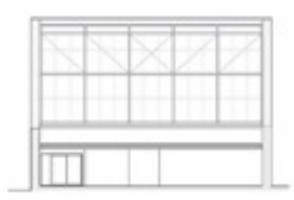

seccion transwersal hacia ef sur

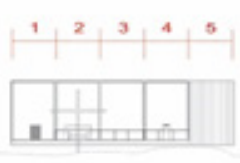

atar_ distribucien
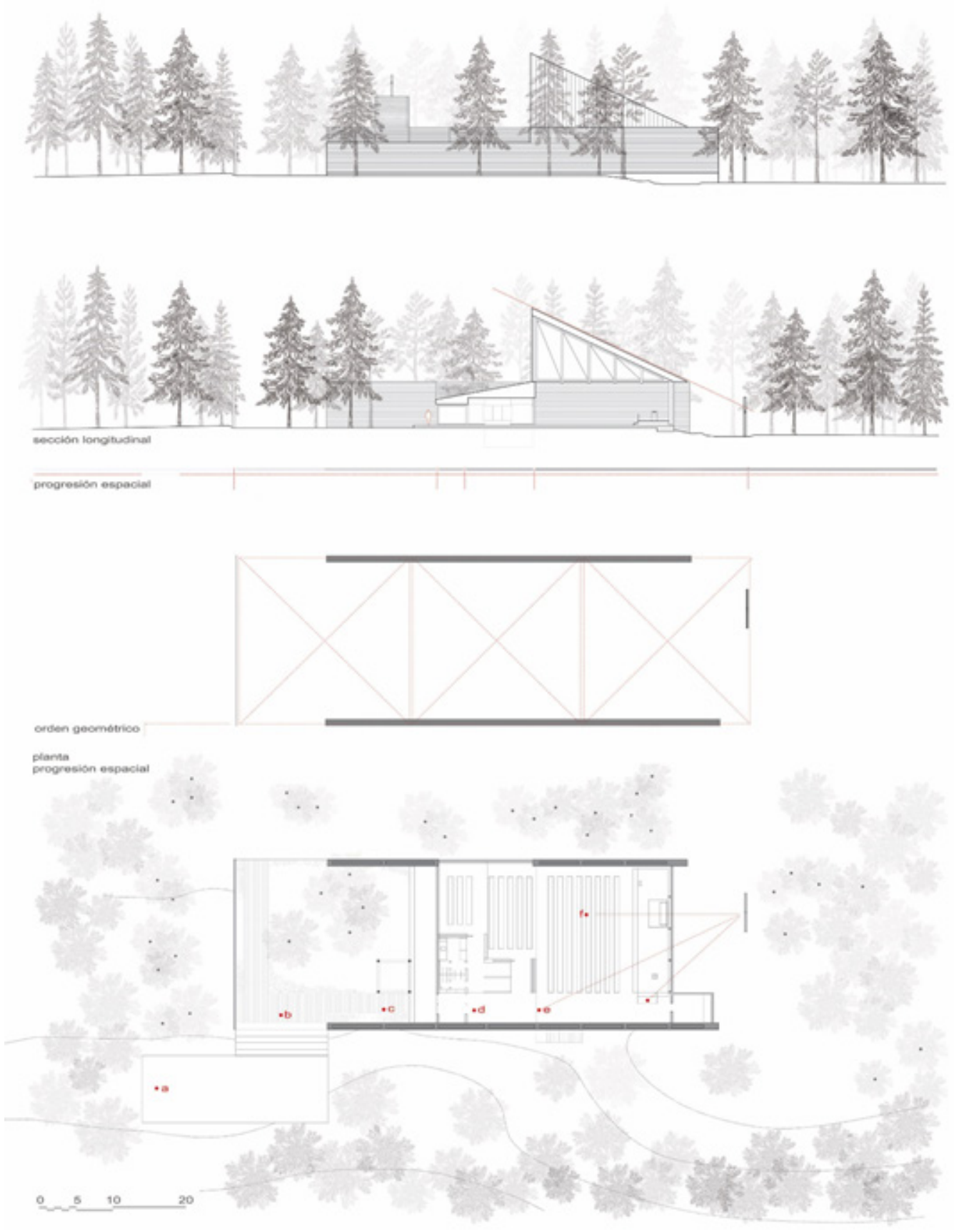

Figura 7: Capilla de Otaniemi. Alzados norte, este y sur, secciones, altar, planta y análisis geométrico

Fuente: Planos esquemáticos redibujados por los autores a partir de los planos del proyecto de reconstrucción de la capilla (1977 y 1978), tras el incendio de julio de 1976, y de la observación fotográfica 
altura y coronándose por finos troncos de madera hasta atenuarse por completo en la empalizada que delimita el atrio, recuerda los distintos cierres de la vivienda de Alvar Aalto en Muuratsalo (1953), que pierden densidad a medida que los espacios se desparraman por el bosque (Figura 8). El cambio de estaciones y el paso del tiempo se expresan en el sutil cierre de la capilla, entre esa "urdimbre vibrante que, como el propio bosque, tamiza la luz y el aire" (López Peláez, 2005, p. 17). El patio está habitado por árboles. De la misma manera que Bryggman mantenía un árbol en la escalinata de acceso a su Capilla, anunciando lo que pasaría dentro, aquí el bosque no se detiene y se introduce en el acceso. El propio campanario exento se formaliza como árboles esencializados. $\mathrm{E}$ campanario que Sigurd Lewerentz había proyectado en 1943 para el Cementerio de Mälmo, a modo de prisma pétreo, perforado por el aire y coronado por una cruz, pudo también inspirar el campanario de los Siren, cuya construcción se alía con la celosía de troncos del cierre (López Peláez, 2005, p. 22).

Dentro del patio, el musgo, cuando no la nieve, nos oculta parcial o completamente la ortogonalidad del pavimento. El suelo presenta distintos despieces, aumentando la proporción de piedra conforme el usuario se aproxima a interior. El acceso debía resultar acogedor y a la vez ex-
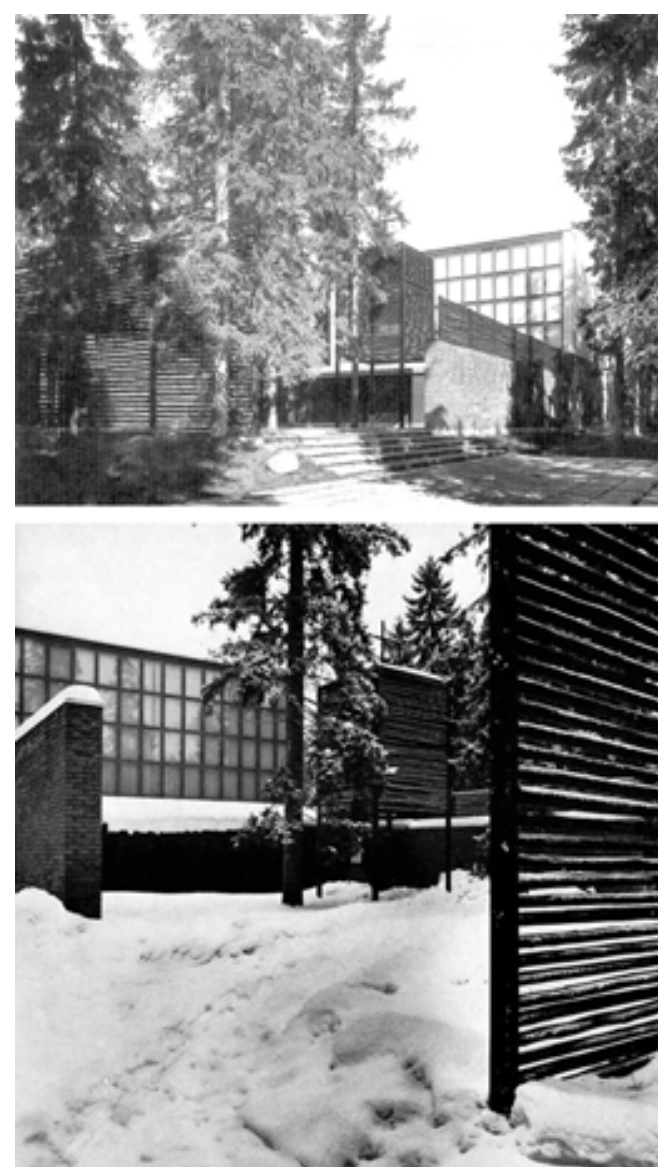

Figura 8: Capilla de Otaniemi. Acceso al atrio y diferentes cierres que definen el espacio intermedio, progresivamente más abierto en la proximidad del bosque

Fuente: Walden, 1998 y Luciani y Puppi, 2009 presar un cierto carácter místico. La fuerte pendiente de la cubierta provoca una interesante sensación de ilusionismo, pero la disposición diagonal de leñoso campanario ayuda a definir la entrada, comprimiendo el espacio que dirige hacia la puerta. Así, los Siren emplearon pequeñas dimensiones en el acceso, resuelto con gran sencillez, para contener los servicios del culto, y lo revistieron de madera oscura, controlando su iluminación. Como subrayaban los arquitectos, debía haber una progresión espacial en forma y luz, desde el vestíbulo bajo hasta la nave alta de la capilla (Norri, 2005, p. 33).

La secuencia se inicia con ese patio que, todavía permeable, pone en situación a los fieles. El pórtico, en sombra, protege a las personas y provoca su silencio. Las dirige en cierta penumbra por el vestíbulo bajo, de 2,30 metros de altura, hasta irrumpir en la luminosa capilla. La cubierta baja afiladamente a lo largo de la nave, hasta la pared de vidrio del altar. Posicionaron el lucernario vertical orientado al sur, de manera que la luz se encauza desde lo alto. Emplearon un material traslúcido para proporcionar una luz homogénea que confiere a la capilla un cierto carácter humilde, casi un aspecto fabril. No permite la visión la luz resbala por la cubierta de madera, los paños de ladrillo y el suelo, también rojizo. Las delgadas cerchas, trianguladas por cables, añaden un delicado ritmo a la nave, potenciado por pequeños focos encajados en los extremos inferiores de los pendolones. Una ventana lateral abierta en los espacios de servicio, en continuidad con la nave, introduce la luz de la tarde tamizada por los árboles (Figura 9).

La Cruz se sitúa tras el Altar, en el exterior, a una distancia que prolonga intensamente el espacio de oración hacia el bosque y consigue que este forme parte de la capilla. Su meditada posición no obedece únicamente a cuestiones simbólicas, sino también perceptivas: es lo primero que observa el visitante al acceder a la nave. Es una cruz desnuda, realizada con perfiles laminados pintados de blanco, que comulga con la nieve habitual en el invierno finlandés y se metamorfosea con el bosque perenne. Abraza a los fieles en el reposo, al final del recorrido.

En la iglesia de San Pedro de Klippan (1963-1967), Lewerentz delimitó el altar con una delgada barandilla y dispuso en su interior una cruz sencilla resuelta en continuidad con el cierre, en un lateral del altar. Así definía el carácter sagrado de la zona acotada (López Peláez, 2005, p. 15). Los Siren emplearon también una barandilla ligera para demarcar el altar, solo elevado dos peldaños del nivel horizontal; sin embargo, la cruz se sitúa tras él, detrás del vidrio que, en principio, parecía delimitarlo.

De esta manera, el recinto sagrado queda ampliado, pasando más allá del cristal, y abarcando el espacio exterior que preside esa cruz etérea; el territorio espiritual, limitado por un lado sutilmente, se difumina en el bosque y se entremezcla poéticamente con los árboles. El lema con el que se habían presentado al concurso, Altair, señalaba perfectamente la esencia de la propuesta. Si Bryggman había hecho del bosque una "nave lateral" para su capilla, los Siren habían construido, literal y metafóricamente hablando, un Altar en la Naturaleza (Figura 10). 

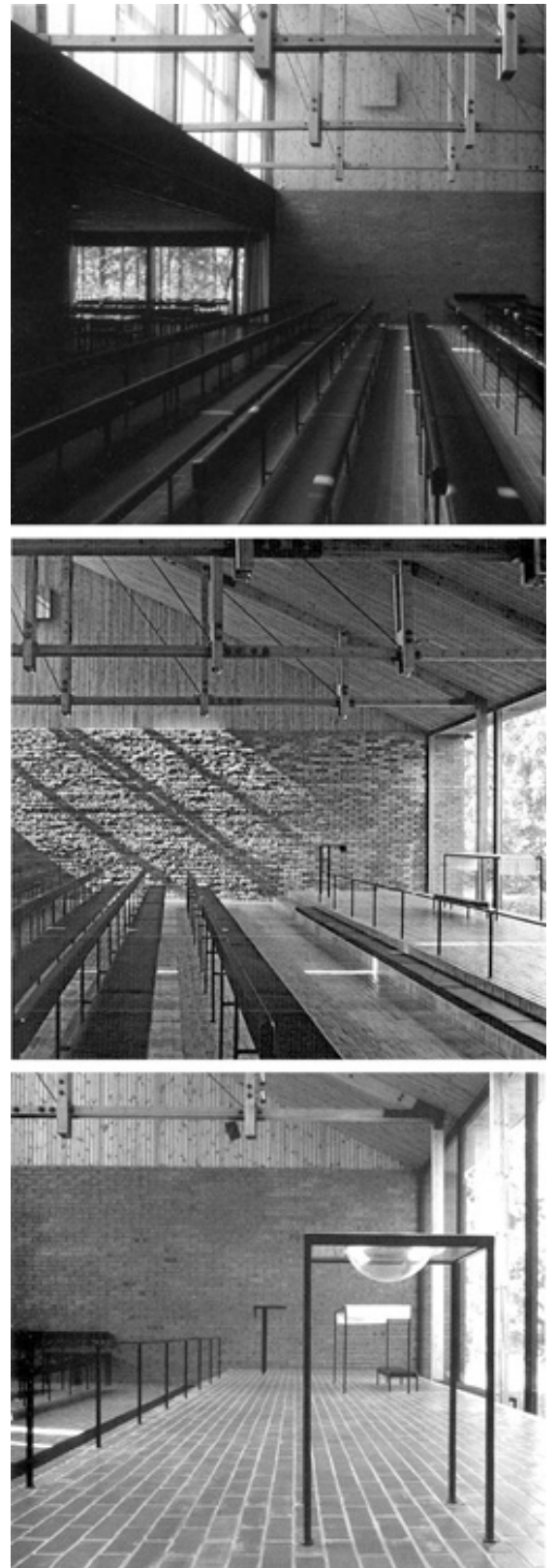

Figura 9: Secuencia espacial de la capilla y parte interior del altar, con la pila bautismal en primer plano

Fuente: Walden, 1998 (a) y Luciani y Puppi, 2009 (b y c)

\section{Lo natural y lo sagrado}

\section{Paralelismos coetáneos y contemporáneos}

Varios proyectos subrayan la relevancia de esta pequeña joya arquitectónica. Pese a su singular sencillez, la capilla de los Siren ha dejado una profunda huella en múltiples capillas e iglesias que, como ella, muestran su altar transparente con una cruz al exterior, en un fragmento de naturaleza. Contemporánea a la Capilla de Otaniemi, Peter Lehrecke construía la Iglesia para el Hogar (Kirche Zur Heimat) para la pequeña comunidad de Zehlendorf, en Berlín (Figura 11). Se elevaba al final de una calle que se iba estrechando y desembocaba en un patio abierto,
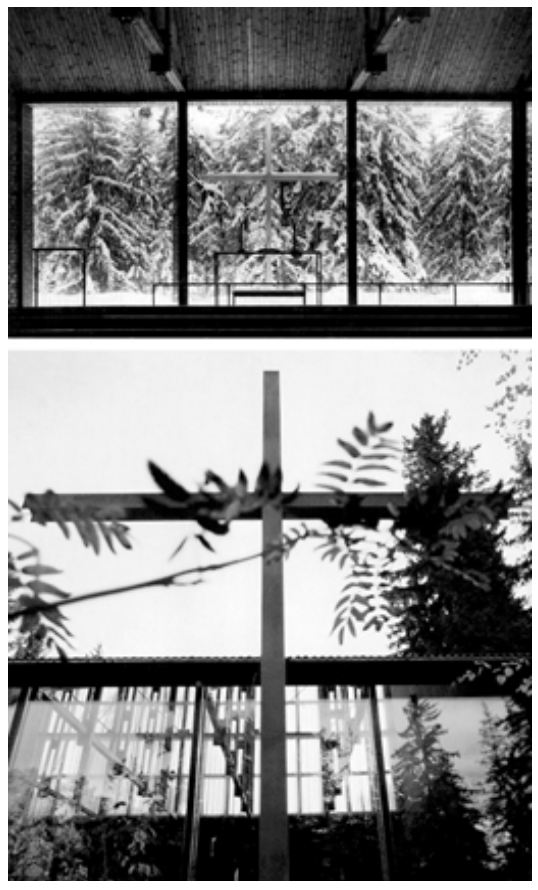

Figura 10: El Altar. La naturaleza como retablo Fuente: Luciani y Puppi, 2009 (a) Walden (b)

que parecía conducir directamente al edificio, subrayado por un esbelto campanario exento, a la derecha, contundente en su volumetría y desnudez.

Salvo por la manera en que se implanta en el lugar, son muchos los paralelismos con la capilla finlandesa. Nacida también de un concurso en 1955, y elegido entre ocho propuestas (una, perteneciente a Frei Otto), el templo

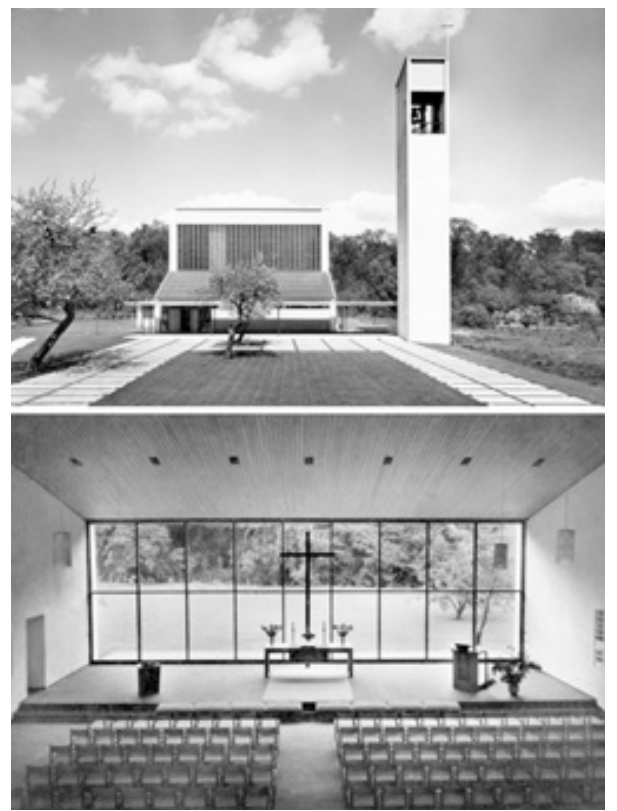

Figura 11: Peter Lehrecke, La Iglesia para el Hogar (Kirche Zur Heimat) de Zehlendorf, 1957

Fuente: Fotografías: Fritz Eberhard Kirsch, Georgios Anastasiades (Schulz 2011) 
alemán pretendía no destacar y crear un espacio litúrgico que pudiera emplearse paralelamente como auditorio para la vecina Universidad Eclesiástica, la actua Universidad Protestante de Berlín. La entrada asimétrica se desplaza a la izquierda y en su lado opuesto se sitúa el órgano. El volumen se construye también a partir de dos muros paralelos y un techo completamente inclinado, que termina en una pared de vidrio. Como en Otaniemi, se busca desdibujar el límite del altar mediante la construcción de una pared acristalada que dirige la vista hacia el jardín, donde se encuentra la cruz de acero. El altar se eleva únicamente dos escalones y un sencillo atril reemplaza el habitual púlpito. En la nave se colocaron sillas en hileras rectas, en lugar de bancos, porque "eran más civiles, menos solemnes"- diría el arquitecto, subrayando la idea de auditorio (Schulz, 2011). El espacio opta por la sencillez, para potenciar la reflexión y el retorno a lo necesario.
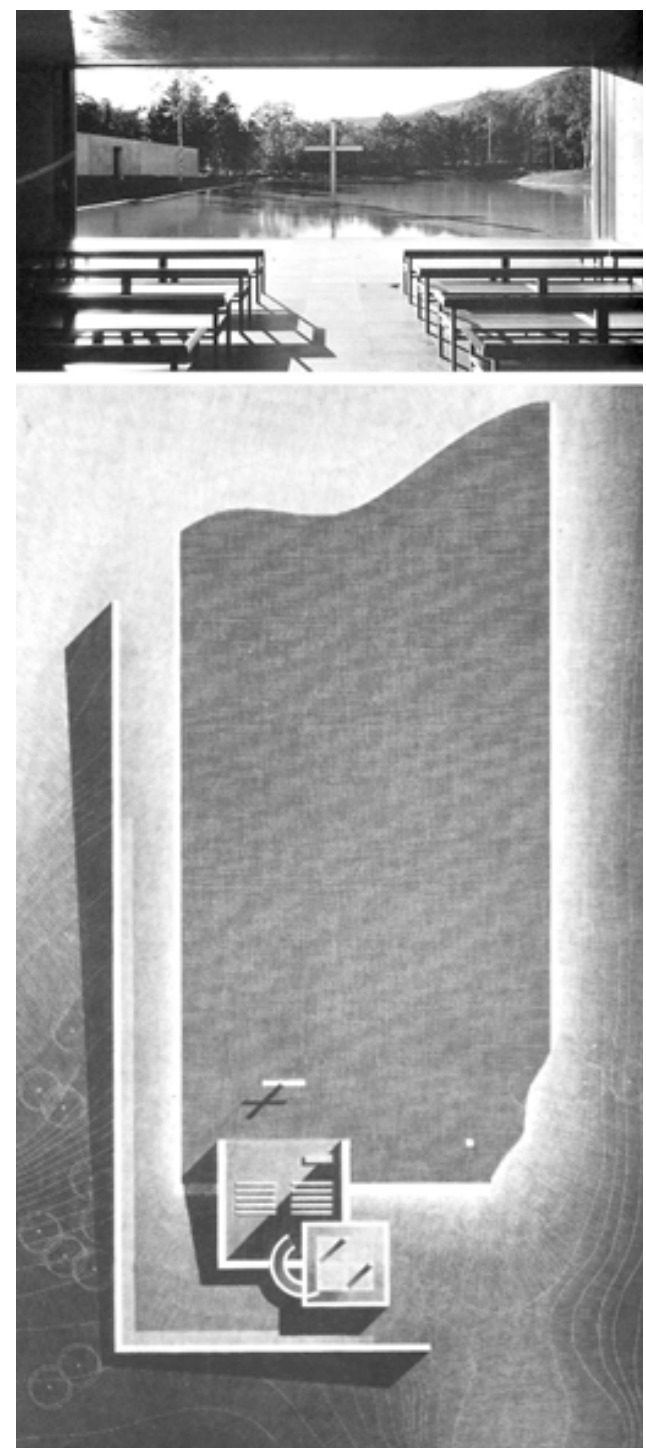

Figura 12. Tadao Ando, Capilla del Agua en Tomamu, 1988. Vista de la nave de la capilla y planta

Fuente: Levene, R. C. y Márquez C. (Eds.). (1994). El Croquis, 44 + 58: Tadao Ando 1983-1993/ Blaser, W. (ed.) (1990) Tadao Ando sketches. Birkhäuser
Algunos templos de Tadao Ando, como su Capilla sobre el Agua (Tomanu, Japón, 1988), entenderán también la relación entre hombre y naturaleza dentro de las mismas claves racionalistas que los Siren. En el sintoísmo la naturaleza y lo sagrado están íntimamente relacionados, por lo que el arquitecto japonés emplea los dos conceptos en su obra arquitectónica de forma indisoluble. Pero a diferencia de la capilla finlandesa, la experiencia de lo sagrado que se disfruta en el espacio interior de la Capilla del Agua no sorprende al visitante, sino que se intuye desde el principio y está en relación directa con los recorridos de acceso a la capilla. Es preciso recorrer el inmenso muro en $L$ que rodea el edificio, y realizar después un movimiento circular ascendente hasta una gran caja de vidrio, para después descender por una escalera oscura y curva hasta llegar a la nave de la capilla. La experiencia culmina en un espacio interior de planta cuadrada que sustituye el altar por una vista del paisaje exterior que recuerda al santuario de Itsukushima, parcialmente sumergido en el mar, en el que un enorme torii rojo rompe la uniformidad del paisaje marino que se enmarca. Tadao Ando sustituye la puerta sagrada por una gran cruz que funciona como símbolo cristiano y sintoísta, que aproxima la imagen de este espacio a la capilla de los Siren (Ruiz, 1995, p. 53). Pero la Capilla del Agua emplea, sin embargo, la artificialidad y amplitud del paisaje mostrado, generando un gran impacto visual que ayuda a representar la dicotomía entre lo limitado y lo infinito. Por ello, a pesar de su innegable similitud con el proyecto finlandés, se aleja de carácter intimista que caracteriza a la Capilla del Bosque, a favor de una mayor monumentalidad (Figura 12).

Nuevos proyectos se inspiran en la Capilla de Otaniem a lo largo de todo el mundo. Así, por ejemplo, la capilla que realiza Jamieson MacCormac en 1990, en la residencia universitaria Fitzwilliam (Cambridge, Inglaterra), o el Templo Budista de Kyoto, creado por Takashi Yamaguchi en el año 2000, emplean la naturaleza como retablo, enmarcando, en este caso, un fragmento de paisaje (Fernández-Galiano, 2002).

También en España la huella de este edificio fue singular Fernández-Cobián explora la sorprendente influencia de la capilla de Otaniemi en las iglesias españolas durante la segunda mitad del siglo XX. Bajo la pregunta "¿Son protestantes nuestras iglesias?" reflexiona sobre el dilema que sufrió la arquitectura religiosa: por una parte, el gusto de los fieles por la imaginería religiosa tradicional, y por otro, el deseo de vincular la nueva arquitectura a las corrientes europeas del momento, más contenidas. En un acuciante clima de renuncia al ornamento que se amparaba en la sinceridad constructiva de la modernidad, se publicaba la capilla de los Siren y, a pesar de pertenecer a la Iglesia Evangélica Luterana, la pequeña obra "quedó grabada a fuego en el subconsciente colectivo", con fervientes seguidores como Alejandro de la Sota y Desiderio Pernas (Fernández-Cobián, 2017, p. 69).

Las Capillas Vaticanas, desarrolladas en el año 2018 para la Bienal de Arquitectura de Venecia, en una muestra comisariada por Francesco Dal Co, es una serie de instalaciones realizadas en el bello paraje de la isla de San Giorgio Maggiore que ponen de relieve el interés que puede suscitar vincular la poética del lugar con el espacio religioso. El Pabellón Asplund, diseñado por Map Studio como homenaje a la Capilla del Bosque del maestro 
sueco, sirve de espacio inicial a los visitantes, para continuar después con diez pequeñas capillas. Proyectadas por arquitectos reconocidos de diferentes países- Andrew Berman, Francesco Cellini, Javier Corvalán, Flores \& Prats, Teronobu Fujimori, Sean Godsell, Carla Juaçaba, Eduardo Souto de Moura, Smiljan Radic y Norman Foster- representan por primera vez al Vaticano en la Bienal, explorando nuevos lenguajes arquitectónicos en la isla donde habita la famosa basílica de Palladio. El objetivo es que el lugar arbolado sirva de espacio para el silencio y la meditación, a medida que los visitantes descubren las construcciones efímeras. La mayoría de las estructuras abren algunos de sus límites al paisaje. Así, por ejemplo, la Capilla de la Mañana de los españoles Eva Prats y Ricardo Flores toma como referencia las Capillas Abiertas de América Latina que, como grandes hornacinas, consisten en un espacio adherido a una nave principal exterior, en este caso, configurada por la bóveda natural de las ramas de los árboles. El portugués Eduardo Souto de Moura opta por generar un pabellón semiabierto cenitalmente, con unos muros de piedra que configuran un espacio primigenio, casi primitivo. Y llevando al extremo la disolución de los límites arquitectónicos, la capilla de la brasileña Carla Juaçaba crea un radical juego visual donde la envolvente del espacio son los propios árboles y e cielo. La estructura de la capilla consiste en dos cruces de acero inoxidable, de ocho metros de largo, apoyadas sobre durmientes de hormigón, que configuran simbólicamente la nave y el altar. Las cruces reflejan la luz del so y el entorno, consiguiendo casi fundirse con la naturaleza (Figura 13).
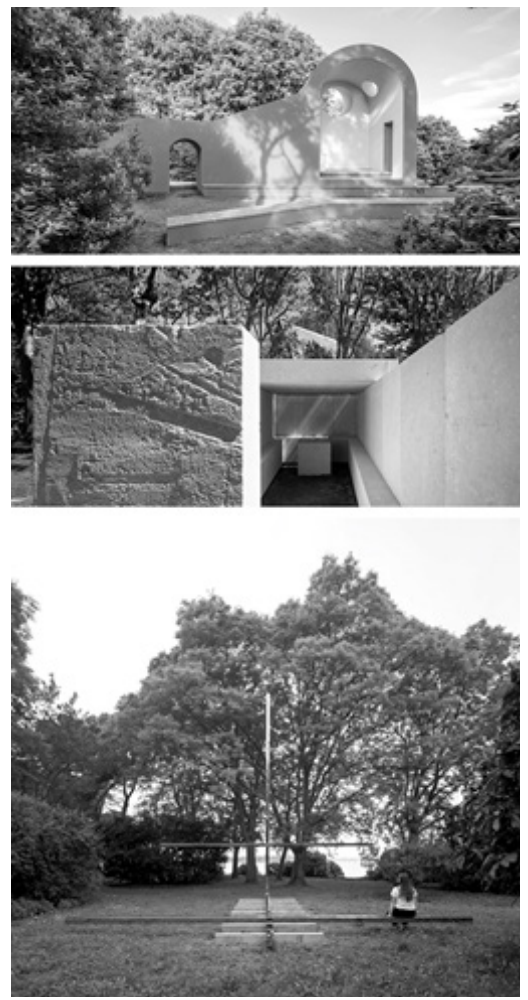

Figura 13: Intervenciones de Eva Prats \& Ricardo Flores, Souto de Moura y Carla Juaçaba. Capillas Vaticanas. XVI Bienal de Venecia, 2008

Fuente: Fotografías: Adriá Goula, en Sebastián, P., 2018 (a) / Alessandra Chemollo en De Juan, A., 2018 (b)/ Alessandra Chemollo en Meri de la Maza, R. 2018 (c)

\section{Conclusiones}

En la evolución del proyecto estudiado, se puede percibir cómo la Capilla del Bosque nace de un lugar específico y se enraíza a él de forma profunda. Los arquitectos aprendieron de Bryggman cómo emplear el simbolismo en la secuencia especial y supieron aplicarlo con una exquisita racionalidad constructiva, que provoca en cualquier visitante una sensación de recogimiento y meditación. A pesar de ser una respuesta específica a un lugar concreto, constituye una lección para otros proyectos que buscan ahondar en significados afines y vincular naturaleza y religión.

Alejada de cualquier vocación expositiva, la Capilla de Otaniemi se arraiga a su bosque. Destaca por su cualidad espacial, por su silenciosa implantación, por la manera en que los límites se van disolviendo para crear una progresión espacial, rica en matices, que finaliza con ese altar abierto: un espacio que sobresale simultáneamente por su cualidad material e inmaterial. Los Siren no enmarcan la naturaleza. La línea divisoria se desvanece: el espacio exterior y el interior se unifican simbólicamente.

En los proyectos estudiados que se inspiran en la capilla finlandesa podemos observar la búsqueda de la meditación y la espiritualidad a través del lugar. En las recientes instalaciones de las Capillas Vaticanas se aprecia, además, una creciente disolución de los límites arquitectónicos; aspecto sugerente y relevante tanto a nivel arquitectónico como simbólico.

Juhani Pallasmaa, parafraseando a Constantin Brancusi, subrayaba: "La simplicidad en el arte no es un objetivo preconcebido, sino algo a lo que inevitablemente llegamos cuando nos aproximamos a la esencia de las cosas" (Pallasmaa, 1999, p.7). Los Siren se aproximaron a la esencia de la arquitectura, y del hombre mismo, con este sencillo templo. Capilla y Bosque, se convertían en Otaniemi en un vínculo idílico, en el lugar perfecto para el ritual y la contemplación, y en un didáctico ejemplo que en el pasado ha suscitado, y lo sigue haciendo en la actualidad, fervientes admiraciones y nuevas referencias.

\section{Agradecimientos}

Agradecemos la amabilidad e inestimable ayuda de Jukka Siren, hijo de la pareja finlandesa, que dio respuesta con diligencia y generosidad a nuestras dudas, tras un encuentro inesperado con la capilla.

También agradecemos a los miembros del grupo de investigación reconocido de la Universidad de Valladolid LABPAP (Laboratorio de Paisaje Arquitectónico Patrimonial y Cultural) la posibilidad de compartir conocimientos e intereses sobre los vínculos entre arquitectura y paisaje. 
Cómo citar este artículo/How to cite this article: Fernández Villalobos, N. y Jiménez Sanz, A. (2021). La disolución de los límites en el espacio religioso. La Capilla del Bosque de Heikki y Kaija Siren. Estoa. Revista de la Facultad de Arquitectura y Urbanismo de la Universidad de Cuenca, 10(19), 21-32. doi: 10.18537/est.v010. n019.a02

\section{Referencias bibliográficas}

Bennet, J. (1991). Sub Specie Alternatis. La Capilla de la Resurrección de Erik Bryggman. En R. Nikula, (Ed.) Erik Bryggman architect, 1891- 1955 (pp. 243-249). Suomen rakennustaiteen museon monografiasarja.

De Juan, A.G. (06/04/2018). Pabellón de la Santa Sede en la Bienal de Venecia 2018 por Carla Juaçaba. XVI Edición de la Exposición Internacional de Arquitectura. La Biennale di Venezia 2018. Metalocus. https://www.metalocus.es/es/noticias/pabellon-de-la-santa-sede-en-labienal-de-venecia-2018-por-carla-juacaba

Espuelas, F. (1999). El claro en el bosque. Reflexiones sobre el vacío en la arquitectura. Fundación Caja de Arquitectos.

Fernández, N. y Jiménez, A. (2014). Un altar en la naturaleza. La Capilla del Bosque de Heikki y Kaija Siren en: D. Villalobos, I. Rincon y S. Pérez (Eds.). Arquitectura, Símbolo y Modernidad (pp. 215-231). Real Embajada de Noruega en España, Universidad de Valladolid.

Fernández-Cobián, E. (2017). ¿Son protestantes nuestras iglesias modernas? La recepción en España de la capilla del Politécnico de Otaniemi. Actas de Arquitectura Religiosa Contemporánea, 5, 66-85. https://doi. org/10.17979/aarc.2017.5.0.5143

Fernández-Galiano, L. (Ed). (2002). Recintos Religiosos. A\&V monografias, (95), 2.

Gil, P. (1999). El Templo del S. XX. El Serbal, Colegio de Arquitectos de Cataluña.

Jové, J. M. (2003). Alvar Aalto: proyectando con la naturaleza. Universidad de Valladolid.

Levene, R. C. y Márquez C. (Eds.) (1994). El Croquis, $44+$ 58: Tadao Ando 1983-1993.

López-Peláez, J. M. (2002). La arquitectura de Gunnard Asplund. Fundación Caja de Arquitectos.

López-Peláez, J. M. (2005). Cruces: La Capilla de los Siren en Otaniemi. En L. Ibáñez (Coord.). Capilla de Otaniemi: Heikki \& Kaija Siren (pp. 9-24). Ministerio de Vivienda.

Luciani, D. y Puppi, L. (Dir.) (2009). The Otaniemi Chapel. Jury Report. En The XX International Carlo Scarpa Prize for Gardens, 2009, The Otaniemi Chapel (pp. 8-13). Fondazione Benetton Studi Ricerche.
Meri de la Maza, R. (11/02/2019). Capilla Vaticana para La Biennale di Venezia 2018 por Eduardo Souto de Moura. TC Cuadernos. https://www.tccuadernos.com/blog/ capilla-vaticana-souto-de-moura/

Norri, M. R. (2005). Una capilla en el abrazo de la Naturaleza. En L. Ibáñez, (Coord.) Capilla de Otaniemi: Heikki \& Kaija Siren (pp.- 25- 34). Ministerio de Vivienda.

Nikula, R. (Ed.) (1991). Erik Bryggman architect, 1891 1955. Suomen rakennustaiteen museon monografiasarja.

Pallasmaa, J. (1999). Una confesión arquitectónica. En Meditaciones sobre el Silencio. Juhani Pallasmaa: objetos y diseños arquitectónicos (pp. 5-7). Ministerio de Fomento.

Pallasmaa, J. (2009). The spaces and images of faith. The Finnish church as an experience. En D. Luciani y L. Puppi (Dir.) The XX International Carlo Scarpa Prize for Gardens, 2009, The Otaniemi Chapel (pp. 93-106). Fondazione Benetton Studi Ricerche.

Quantrill, M. (2005). Finnish Architecture and the Modernist Tradition. E \& FN Spon.

Royo Márquez, M. (2014). Pietilä. El proyecto de Dípoli. [Tesis de doctorado, Escuela Técnica Superior de Arquitectura de Madrid]. http://oa.upm.es/33124/

Schulz, B. (2011). 1956-57... In die Jahre gekommen Kirche Zur Heimat in Berlin-Zehlendorf, $d b$ deutsche bauzeitung, db. https://www.db-bauzeitung.de/db-themen/ $\mathrm{db}$-archiv/in-die-jahre-gekommen-kirche-zur-heimat-inberlin-zehlendorf/

Sebastián, P. (05/06/2018). La Capilla de la mañana de Flores \& Prats Architectes para la Bienal de Arquitectura 2018. Metalocus. https://www.metalocus.es/es/noticias/ la-capilla-de-la-manana-de-flores-prats-arquitectes-para-la-bienal-de-arquitectura-2018/

Siren, H. (2009). Wood, Nature and Architecture. En D. Luciani y L. Puppi (Dir.) The XX International Carlo Scarpa Prize for Gardens, 2009, The Otaniemi Chapel (pp. 26-28). Fondazione Benetton Studi Ricerche.

Tucci, F. (1994). Natura e memoria nell'architettura finlandese/ Raili e Reima Pietilä. Parámetro, (205), 61- 67.

Walden, R. (1998). Finnish Harvest. Kaija and Heikki Sirens'Chapel Otaniemi. Otava Publishing Company. 